\title{
Un caso de Septicemia Puerperal por Neumococo
}

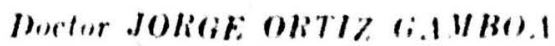

Cirrude ha sida of progreso aleanza. do en los viltimos años en lo yue se re. fiere al eonocimiento, diagnívtivo, profi. laxis y tratamiento de las diversas in. feceiones que se pueden presentar du. rante los días correspondientes al puerperio ocasionadas por agentes patóge. nos cuya puerta de entrada está representarla por la herida placentaria o por las soluriones de contimniclad patoló. grieas, tales remo los desgarmos cervian. Jes, vaginales y perineules.

Sin edmbargo. a pesar de todos esos adelantos y de los miltiples inidarlos que hoy se observan en la remeión do los partos no dejan de hacerse pressen. tes algumas formas de tales infereiones.

Tiene por objeto este estudio el de dar a ronower un ease de Septicemia

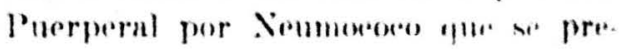
sentí en mat enferma de. Servioin de

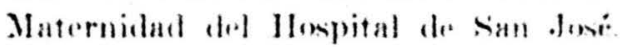
siemole do gran interés no síle por la pora freenenein de este mirerorganisno como agente eausal de infereviomes puer. perales, sino también por ser ponible In deseripeion de la sintonutologín loj rнaslro elimieo y de los magnificos re. siltarlos qua se abtuvieron lobil la tera. péuticn emploradu.

Desplion de haber revikato las enta. distiens ale los hospitales de San oluas de. Dios, de sen José, de In Flinirn de la Provideneia. axi como la mayor parte de la liferatura Obstétrica, podemos concluir pue es la primera vez que se reporta un caso comprobado de una Septicemia Puerperal, ocasionada por el Nirumoronet).

\section{HINTORIA CLINICA}

llinfuriat numero 2151.

l'sbellon finillermo fiomez.

Nombre, A. O.

Fial. 25 años.

Ferha de entrada: 30 de mero de 1951

lugar de origen: Ia Palma.

lingar de proedieneia: Bogofá.

Numero de emburazos: sermolizes. intite.

Antenesentem herediturios: sin in. purtamein.

Anferententes patolégiens: paluctiomo. hase dos gñm.

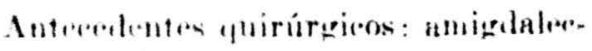
fomia, hace siete años.

Antreerlentes ginemológieos: menar. inin: ? Ciclo: $30 \times 3$.

Anteredentes obstétrieos: In embarama termino eon parto expontioneo, foro vise? puerperio normal.

Embarazo actual: ferba de In ciltima menatruarion: 30 de abril de 19.5). 
sirvelo: a termmo.

Examen general. Piel: sana.

Sistema eireulatorio: pulso: iti por minuto.

Tensión: Mx. 10, Mn. 6.

Ruidos cardiacos: normales.

Sistema respiratorio: normal.

Sistema dipestivo: normal.

Sistemat qúnito-minario: normal

Talla: 1.50 metros.

Poso: (it,5 kilus.

Errimenos de Laberatorio. Reargen serológriens: megativo.

Radioscopia pulmonar: 1 " gat i vo para TSB en evolurión.

Examen parcial de orina:

Color: ambar.

Aspecto: turbio.

Densidad: 1014.

Reaceión: ácida.

Pll : 6.

Albúmina: huellas.

Sedimento: células epiteliales $x \mathrm{x}$.

Leucocitos $\mathbf{x x x}$.

Examen obstétrico. Senos: normales.

Pezones: prominentes.

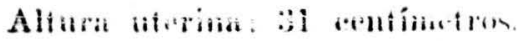

Embaraze inime: si.

Presentarión: afólica, vertien.

Enrajaula: si.

Ponicion: izquerda.

Variellad de posiegon: anterior.

linidom rardianos forales: pention.

Tacto vaginal: $30-1-31$ - 11.15 a. m. C'uello blando, berrade, dilatarión complata. membramas rotids.

Parte. La enferma inieic el trabajo espontáneamentr a las 4 de la maiana. del 30 de amero. Cuando llegro al hervi. eio (11 a. m.) presentaba rentraneiones berinas de intemsidal normal flue ore smerdian esta minuto y medio rour una se nalaba muy atallzato a lat pmone. ique se estaban verificando los primeros tiempos de la expulsión fetal; en estas condiciones se ordenó el traslado inme. diato de la enferma a la sala de partos. no alcauzando a ser pasada a la mesa. ya que el feto fue expulsado en la ramilla a las 11.20 a. m., orasionando 1 , desgarro perimeal de primer grado.

Tante ol nino conos la matre fueron atemdides por ol médien-interno dol

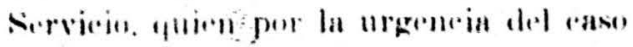
no tomó las dibidas presaneiones de asepsia. El ulumbramiento se verificó a las 11.25 a. m.. expontineamente y en seguida se hizo ba sutura del desgarro perineal. Como tratamiento después del nlumbramiento se le aplié una ampoJleta de Ergotrate vía intramusenlar.

Hora de sulide de la sala de trabajo. i1.40 म. m. Tension: $11 \times 6$.

Estade di la paciente duroute las se is primerns horas. Satisfactorio. Ketraceión uterina: normal. Pulso: so por minuto. Tension: Mx. 101\%, Mn. 6.

Pucrporio. 31 enero. Senos: bien.

Involueion uterina: is antimetros.

loquios: sungrantes.

Tratamiento: liclo Eragotrata Tbl. nimere 2. Sulfuliagina.

Thl nimero 30, Tomar don emla roa tow horas bañe exterto.

10 febrero. Senos: bien.

Involu.iin uterina: 12 eentimetron.

bonuios: sangrantes.

la enferma se anellentra an marcado entade de sopor. Sin ambargo, relata que en las primeras horas del lian pre. sento un violento asealof ríc gaompana. do de intensal eefatalgia y dolores éseos peneralizades. torlo lo rual fue segnido de gran sensarión de ealor y sudora. i ion profusa. 
exament le la enfermat y se enelaentra lo signiente:

Insprevinin: rara mụ reongestionada, •onjumfivas inyertadas, fotofobia. Piel limmeda, ealiente. Ina ligera menejien earacterizalat por pepucins manelus

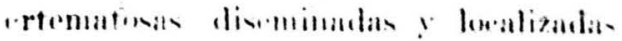

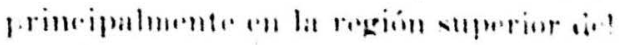
tiriat.

Pinpreratura aniar: fll grathe

Sistema rirrulatorio: pulso: 1:21 pus saciones in el minuto.

Tensiin arterial: Mx. 10, Mn. 6.

Ruidos cardiacos: velados.

Sistrma respiratorio: 30 respiraniones 1.n el mimito.

11 examen pulmomar no se emences: 1 ran sigmos patológriros.

Sistema digestivo: lengula sereat. sit lurral en el eentro y lisa y muy roja en los bordes. En al examen del abdo. men. silo llama la atemeion el ifero ya. sobrosal, 1' remtimetros por encima del mols, en involurión normal. El baz" no s palpable ni perentihlr.

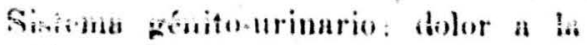
presion an los pumfos remales pusterie

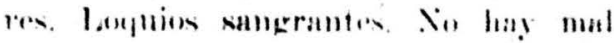
olor.

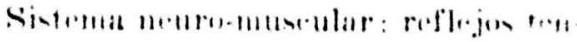
dimonom exageratus.

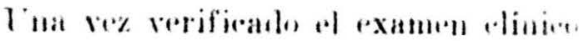
de la enferma se solieitan algumes . manes de laboratorio.

Pere, en las horas de. la turele. s. agmoliza Ia sinfomatolugía do la parion. to: Ia temperatura aleanza tl grades, al pulss tiene unn freenenein do Ifu al minuto. In tomsion artorial so inalua all ! In maxima por 6 la mininus. is respiraciones en el minuto, ol extgdo

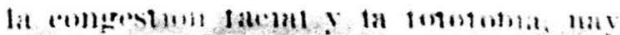
alaten nasil.

Sobre nmbms mejillas se emenentrum man.h.s violineas, simétriens, de tipa m. urlatiniforme. In erupeion qu. enem.

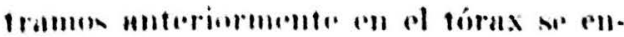
clinera mas disemimalo en forma de gramile man.has eritematosas que des-

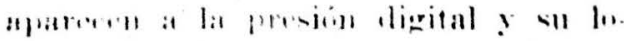
alizaren :

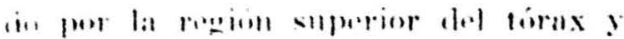
rmello, por el dorse de las manos y re. gioin pesterior de los antebrazos.

1.0 yue mas molesta a la enferma es la intrisa eefalalgia que se hace cada vez. mis aguda.

Antr este cualro sintomático se piensa on ma posible infeceión puerperal I entolles se presicribe al siguiente tratamientu: penieilina 1.000.000 11. para divolverlas en 1.000 (c. c. de suero dextrosado al 5\% en arun destilada y aptiearlo via endovenoma a raxiu de 20 gotas por minnto. Sulfarliazima, tнbletas mumero 2 cada ruatro horas.

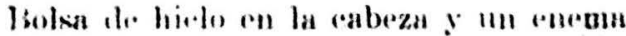
evaenalor gh que la enforma presenta tambión eanstipación.

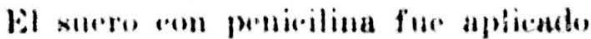

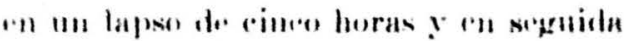

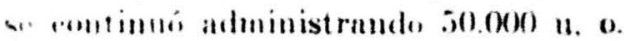
de. Penicilian eada tres horas y Estrep. p.ruirina 0.25 gramos cada ellatro horas via intrammu-ular.

I fobrero. La enferma ha regecions. … algo ill sill estato general.

la truperatura so halla en 40 grados. lil pulso: 10x en el minufo. Tensión s.rferial: Mx. 8, Mn. li. Ја ерfulalgiн .ontinúa. El estado d. sopor la dismi. muido. Ias manehus excurlntiniformes d. In eara s. han ido extendiendo ha. cirndos. más nitiolas. Ademín de Ins 
manchas ermematosals ylue se cmenomtran en la región superior del troneo. dorso de las manos y eara posterior de los antebrazos ha aparecido 11 n nevo elemento earacterizalo por pápulas muy pequeñas que se localizan en el centro de las manelas eritematosas. Xo hạ prorito. Si borran a la presion.

Resultades de los eximemes de lailoratorio:

Hemoparaisitos: mo se enemtraron.

Cuadro hematioo: hematios: mormal.

Hemoglobina: 13.5 gramos.

llematocrito: 44.

Jeruocitos: 13.100 por $10 m .:$.

Eosinófilos: 0.

Basofilus: 1.

Mirloritus: 0 .

Jureniles: ().

C'ayılo: ?.

Segmentades: 81.

linforitos: $\bar{i}$.

Monoritos: :2.

Sedimentación globular: 2t mm. an una hora.

Examen parejal do orima robor : amarillo.

Aspurto: ming torbio.

Demsidad: no uleanzó la muretra

Roareicin: áricla

l'H: (i.5.

Alluminal 117 mers

filunas: It mares.

Acetoma: 110 hay.

l'ignentes biliares: mo hay.

Sedimento: células epituliales $\mathrm{xx}$.

leneoritos $\mathrm{xxx}$.

lionitos: no hay.

Homatios: no lay.

Hareferias: aloumbanter.

C'ristakes de sulfas $\mathrm{xxx}$

Cilindros: granulowes.

Baterianos abmolantes.
Alzut

primer diagnostice y se piensa entonces que toda esat sintomatología corresponde a una posible sensibilidad por las sulfas y se ordena suspender el tratamiento a base de antibióticos y en rambio so prescriben antihistaminieos. min purgante areitono y stlero dextrosiado, vis reldeventesa.

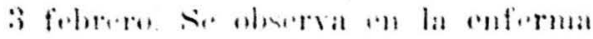

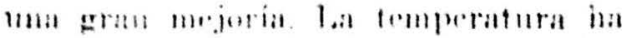

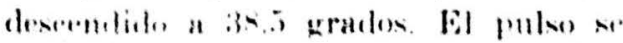
b:alla $1 / 11$ r. arterial lo por fi. In cofalalgia ha ce. dido y es menos infensa. El estacio de sopor ha desaptaresido. La involución uterina ex de ! rentímetros y los loquios contimian sanerantes sin presentar mal olor. La erupeión eritematosa es menos marrada, mo se ha sermido extemdiendo pero en rambio las papulas han evolucionado a menonas pustulas dismoma. das, indoleras, que se lowalizan prefo. rentemente en el dorso de las manos y region poserior de los antebrazos.

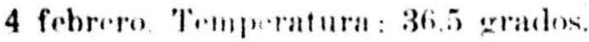
lulso: si .n ol minutu. Tension arte. rial: 10 por do lia sintomatología gene. ral regresa rapilamente. In enferma so romenotra trampuila y mo se queja de molentin ol:rumn

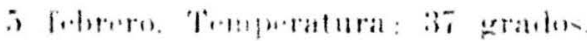

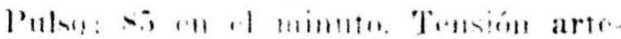
rial: In for fi Involubion merina: mormal. loguios serosanguinolentos.

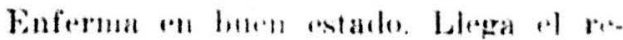
sultale del hemoneltivo: positive para

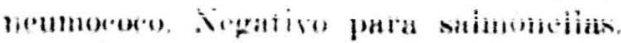
Se ordena entourer m rxumen barte. riologico del entenido de las pútulas.

6 folorero. Tomperatura; 37 serados. fulse: 90 an mime. Tensión arte. rinl 10 por ti. Eulorma on magnifitas

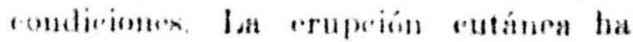


dejando una ligera descramación.

7 febrero. Temperatura: 37 gralos. Julso: 80. Tensión: 10 por 6. So ha suspendido toda medicación. El resul. tado del examen bucteriológieo del con. tenido de las pustulas da position para ol nelumorero.

S folorero. Siale la enforma del hom pilul

('ausa: mejorin.

Estade general de la enfermit: vatio factorin

\section{COMENTARIO}

Hemos reportado la Historia Clínien de in raso que presentó una de las infecciones puerpirales más grases o sea la Septicemia Puerperal.

Una ve\% remnidas las manifestariomes rlinieas de la enferma ron sus anteee. denter silo pudimos llegar al diagnóntice positivo de exa entidad patologiea mediante of hemecultivo que meproduje colonias de $11 \mathrm{n}$ mirroorganismo enusal muy raro an estas formas de infeceio. nes puerperales: ol neumoroeo, que luw. romprobado mís tarde por il extudió bacterioligieo del contenide de las lesiones cutinleas.

Ya determinada la forma die infer. rión y su agente etiológien, sille nu. quedaba por averiguar el punte de pas. tida y el mecanismo que habia seruide. Por informes verbales del médien-inter. no que atendió a la paciente en el mo. mento del parto pudimos concluir que él constituía la furnte exógeua en ia produecion de la inforecion in que se tratala do 111 portador sano de exe

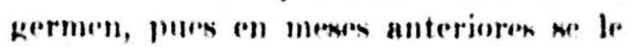
labía practiendo orasionalmente un examen bacteriológico d. In rinofaringe, encontrándose un prodominio mar. pos microseópicon sin estar producien do lesión alguna.

Esto viene a coincidir eon to expurs. to anteriormente en In historia, o men. la atención de urgenein de la enfermu en el momento de expulsion fetul, sin observar los cuidados de asepsia que son d. rutina en estos rasen y entre lom cuales sobresale ol uso de la masearilla

Asi pulimos romeluir que se trataba de 11 a infeceión purerperal exógena. yue ex la mis comin. cuyotegente etiológico fue transportado al conducto genital por el mismo médico y la puerta de entrada estaba constituida por el desgarro perineal que se produjo en la enferma. Una vez alli localizado el neumococo exaltó su virulencia meciante circunstancias especiales aclquiriendo un pran poder invasor en el torrente cirenlatorio produciendo un elladro elínico grave all el ellal predomimaban los sin. tomas gemerales pues los loceles eran muy Ps'hoses.

Queremos llanar la atencion al hecho de que presentando un cuadro clínico lan grave y de pronóntion mus aevero lubiera cedido rápidamente con el tra. tamiento establecido on las primeras 24 boras. Esto nos indiea gue "l germen ransalute de la infereción cra sensible en nlte grado a lon bactoriotatices y anti. bióticos de tipo Penicilina y Sulfadin. rina, ун que de la Estreptomieina sólo se le administró 0.50 centigramos, pues se suspendió ess medirarion al dia niFuiente al cambiar la orientacion del diugnistieco.

Como medida profilática debemon tener en cuentu, ul presintur este inso. que, conociendo la eptielogía de la in. feecion puerperal, las caunas que on olin predisponen, la formn de llegar los mi. 
tales. el modo de impectir la exaltación de la viruleneia le los que alli viven y de aquéllos que son apartados desde el exterior. la manera de ammentar las propiedates baterividas del organismo. antes de que la infereion aparezara, debe ser objeto de enalquier tratamiento profilartien flue fiembla a impertir la apat-

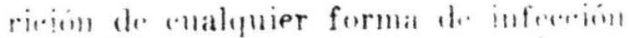
[merper:i].

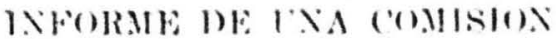

Para dar emmplimionto a la impor-

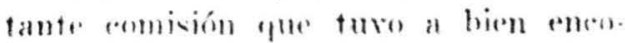
mendarmos la Mesa Direntiva. hemos estudiarlo muy atentanente la intere. sante historia elimina presentadit por el

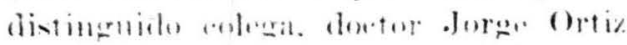
Gambuat y que llevat pur titule "ln ease d. Septionial l'urpural por Xeumoxesen.

Fl dextor Ordiz (immbor hace hinea-

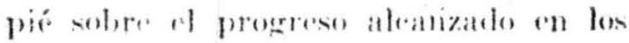
bitime anos, en lo reforentr al romorimienfo, diagnostico, enpofilaxis y tra. trmienfo de las diversos infereiones que a porelen presentar dumate los dias

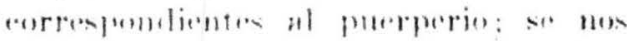

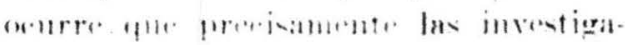

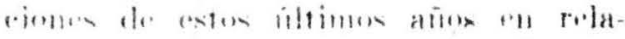

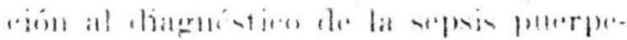
ral moly pexes se hat allelantade. debido H) Iniserestmente serplate fratamionto

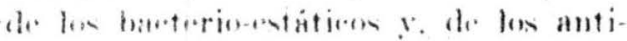

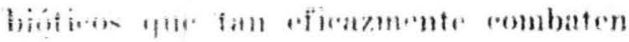

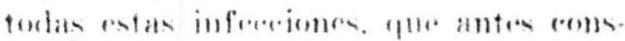

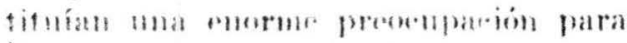

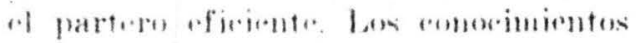

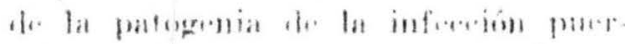

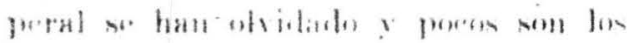

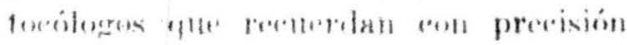

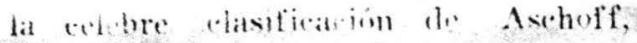
rillen distinguia:

a La antoinfereion espontinra. cin la rual lon mirrobios vaginales fenclogenos) asejemelen espontanedurente a la matriz.

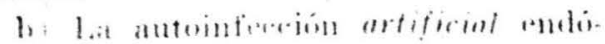

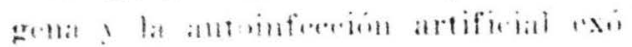

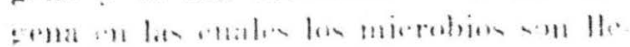

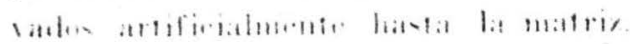

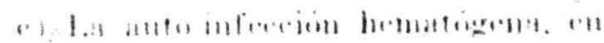
b matrix, por vin somglimea. por menomis. mo die metastasis de otro promese aenào, verbigracia मngima o nemmonía.

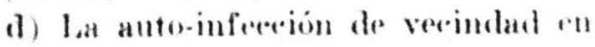
la qui los gérmenes llegan a infectar por simple eontimuidad murosa o por los linfitions

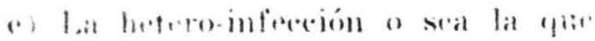

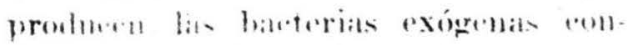

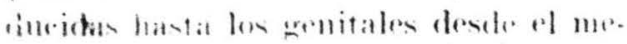
(io) exterinr.

Por ate bltime meranismo fue romo

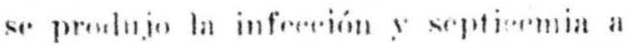

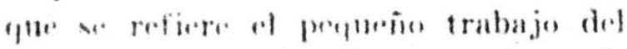

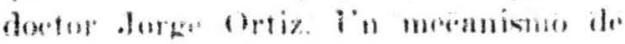

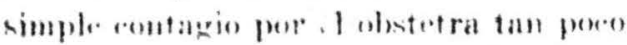

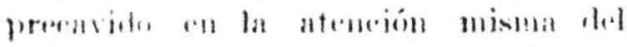

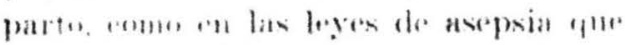

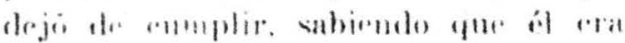

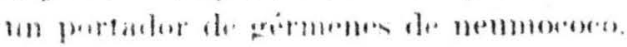

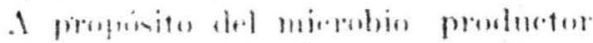

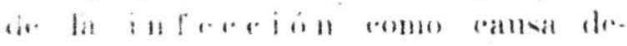

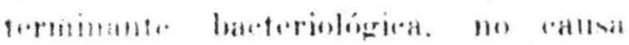

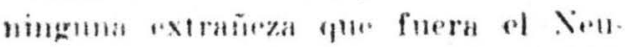

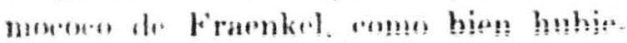
ra polite s.r. ol barilo de. Eberth, el de Nieolaier " ol de l'feiffer anngare en

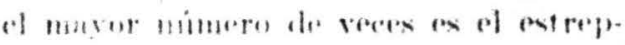

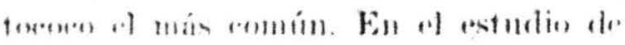

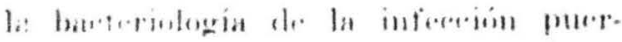

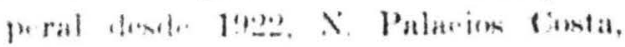

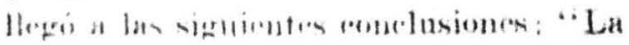


infeceion la puede detreminar coual. quier gerentell. pero sobre todo los del "rupo llamado séptico; igualmente ufir. maba el mismo antor ylle la presencin del germen en la sungre no justifien diagnestien de septicemin. silln qute a

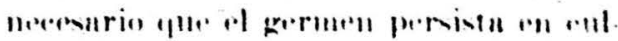

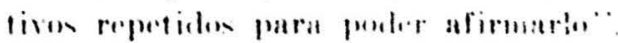

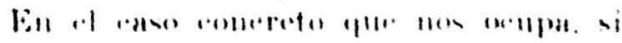

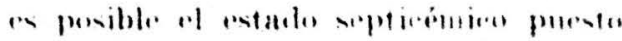

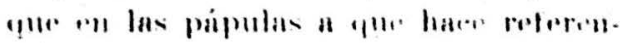
ria la historia y que evolucionaron H

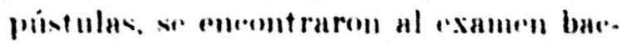
epriolónien numerosos nemmoeomes. bse Eritema lo podemos definir descle el binto do vista anatomopatologicos: cr. tema saprofitario.

la interesante historia elinica pre. sentada por el cloctor (Ortiz fiambos. a la Soriedad de Obstetrieia y fimereole.

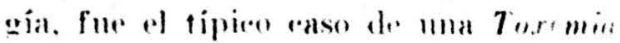
Puerporal. eomoricla también eon el nombre de Nopremia perepral de Itunran, caracterizada eomo el resultude d. al artividad sнprofitaria

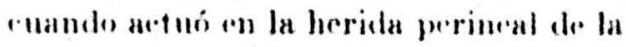
parturienta. y que descraciadamento pasi por vín hematógena n eouvertirs. in siretiormin ale nemmoro.

En lo reforente a sinfomafologia eli. nira de dirlon toxemia. Hama la aten. riin la pora 0 mola semsibilidad merina y la correcta involución de lat misma. sin loguios féfidos, ni signos de proseses encometrial, dol parametrio ni los anexos uterimos, es decir. la allsemeia fotal de sintomas locales in los genitaine allugue no sabemos por el relato histórieo heeren del resultado final do la suturn perineal.

Fs de anotar romo sintomas perulia. res: Ia cara congestionacla. In fotofobia. la piel himuola y ediente y In rrapeion enraeterizarla por pequeñus manchas erifenatomas diseminadax y localizadar priserpalmente all la regien stepriog li.l hirax y loégu en las manos .Mle. mas. manificela el ponente. hubo dolor n la presion en los puntes renale for. teriores ene temperatura (le 4l) grade.

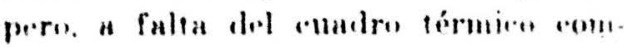

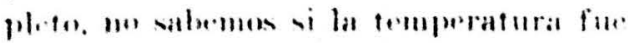

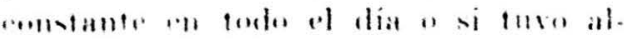

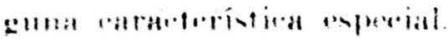

Al ramen d. arima, presente albi. mima of. 117 miligramos. élulas epite. lialis y lenconeifos. Bacterias abmolantes (no presisn de qué tipos. Cristules de sulfas y eilindros gramulosos.

En eambio el coadro hemátieo llami In ateneión la lencocitosis de 13.1(K). In lemorlobina de 13.5 Hematorito: $f i$. limforitos 7 y la sedimentacion de 24 ma: in una hora.

Tratamiento. Dice la historia literal. mente que " Hnte una pesible infereion pilerperal se preseribe el signiente tra.

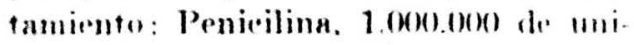
ciales para disolverlas en 1.000 e. e. de

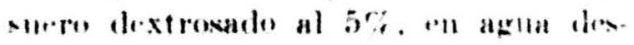
tilucla y apliearlo por via embosinoma. "razon d. 20 gotas por minuto. Sulfa. diazina, 2 tabletas coula ruatro horas: ledan ale hielo en la rabeza y 111 enema andualur. Al terminar ol sirro s.

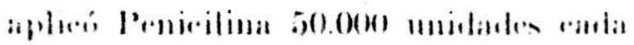

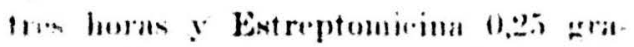
mon rata reatro horas, vin intra-mus(rinlar.

('omo presentara signos de intoleranc ia por las mulfas. Ias suspruden y proseriben antihistamínicos, 11 pureant. neritoxo y suero dexfrosudo. por vía ell. dovenosh".

Este tralamienfo fur instituino xin

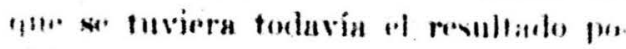
stiva pura nemmocoso quer quiere dereir, que ol mismos trata. wiento lubiors servido para eualquier 
clase de acente prodinetor de la sepsis, ya fuera el estreptoroco, estafilocono "1 neumococo y hasta para el gonococo.

La enferma mejoro y a los nueve lias salió del Hospital por mejoría.

No queda otro eamino que felieitar muy ealurosamente a quien instituyo el tratamiento en beneficio y ugraderimienter de la pariente. Congratularnos por el hallazgo oportmon del netumonow יla sangrer y en las lesiones de la piel. V, por lo que respecta al elestor .Jorge Ortiz finmboa, felicitafon muy de veras, for el aeopio de su importante historia.

Para eumplir con los estatutos de la Socjedad de Obstetricia y Ginecologia. nos permitimos poner en eonsideracion d. la Sociedad, Ia siguiente proposirion de vuestra comision! :
La sociedad de Obstetricia y limecología, después de oír el comentario al trabajo presentado por el doctor Jorge Ortiz Gambon sobre "T'n caso de septicemia puerperal por neumococo". para el ingreso a la Sociedad, se declara satisfecha de su importante colaboración. pide que la historia clinich sea publicata en la Revista, junto ron st comentario y lo propente romo sorein afiliado, manifestumblole sll desen para que continia por el semdero de la in. vesfigarión que ha inicialo. рати qше muy pronto y con un nuevo aporte científico, lo contemos como sorio de nímero de esta Corporación.

Vuestra Comisión.

\section{Campaña contra el Cáncer}

Bogoti, rgosto 20 d. 1!).1

Señor l'rofesor

Hernando A пиун lán.

l,a rindad.

Apropiado dextor y amigo:

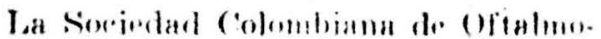
logía y otorrinolaringologia. de (obombia, ha querido iniciar йя eampana

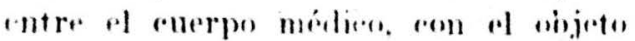
de velar por el diaguóstico presez de las lesiones meoplasions de la laringe.

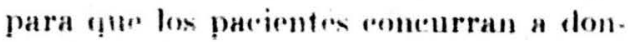
fir el mérlico esperialista o a los erentro, Appeiulizalos, a sil debido tiompon se purde practicar un tratamiento plie ofrezsa 11 poreentaje alto do curarioin.

Hemos querido también, मl mislla liempo. informar a nuestros rolega sobre las causas mas frecuentes de a.. guer. eon el objefo de romtar rom su valiosa eoluboración y dismimuir en exta form las imụmerubles pérolidas de in visión que se pueden evitar haciende iII trataminento aderuado n su debiclo fiempos.

Para llemar extos fines rogamos a meterla so sirva prestarmos sll valiona rolaborarion. publicanto difumlida y leida Revistu los enquemas adjuntos. en las fechas y lingares que usterl ensoidere más indiedades.

Mucho agraclecerímos una colabora.

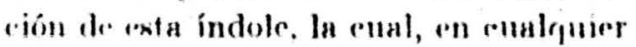
momento sabriamos eorrexponder on la miкma forma.

Aroptimino amigo y rolegn,

carlos A. Cleves Cicalón Presidente. 